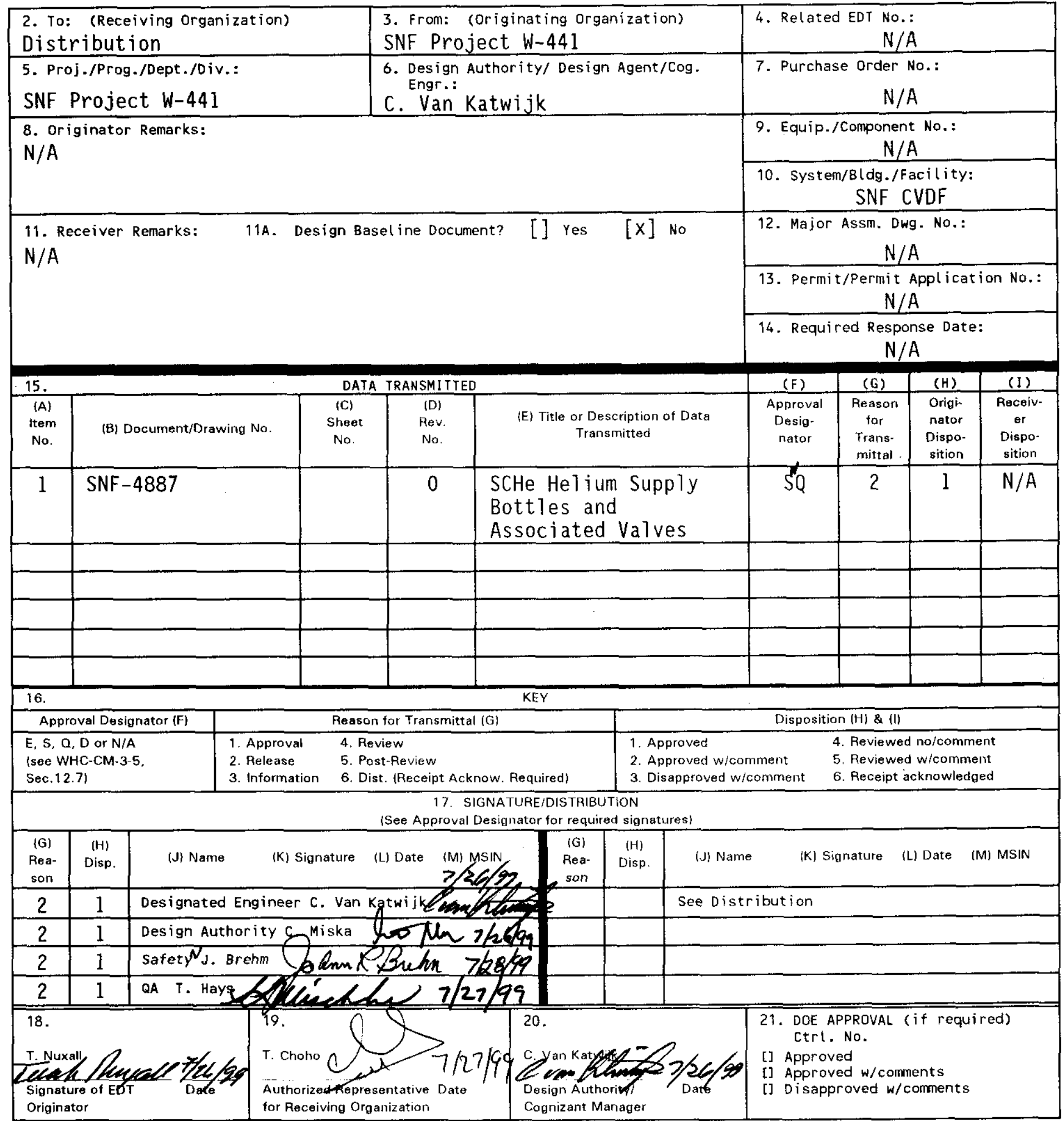

BD-7400-172-2(05/96) GEF097 


\section{SCHe HELIUM SUPPLY BOTTLES AND ASSOCIATED ISOLATION VALVES}

Carl Van Katwijk

Numatec Hanford, Richland, WA 99352

U.S. Department of Energy Contract DE-AC06-96RL13200

EDT/ECN: 626322

UC: 620

Org Code: $2 \mathrm{G} 300$

B\&R Code: 39EW40400

Charge Code: $105559 / \mathrm{A} 000$

Total Pages: 13

Key Words: SCHe Helium Supply Botıles and Associated Isolation Valves

Abstract: SCHe Helium Supply Bottles and Associated Isolation Valves

TRADEMARK DISCl.AIMER. Reference berein to any specific commercial product, process, or service by trade name, trademark, manufacturer, or otherwise, does not necessarily constitute or imply its endorsement, recommendation, or fivoring by the l hiled States Government or any agency thereof or its contractors or subcontractors.

Printed in the United States of America. To obt:ain copies of this document, contact: Document Control Services, P.O. Box 950, Mailstop H6-08, Richland WA 99352, Plone (509) 372-2420: Fax (509) 376-4989.

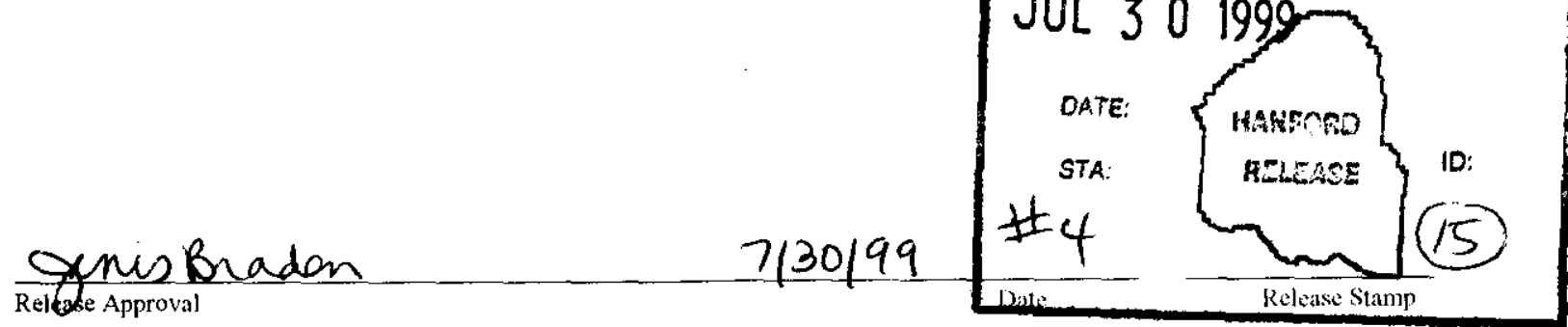

Approved for Public Release 
Commercial Grade Item Upgrade Dedication Form

ECN No. NA _ CGI No. CGI-SNF-D-13-2-P5-050

SNF-4887. ReN 0

Titie: SCHE HELIUM SUPPLY BOTTLES AND ASSOCIATED ISOLATION

VALVES

\begin{tabular}{|c|c|c|c|c|}
\hline \multicolumn{5}{|c|}{ Section 1 Part Information } \\
\hline Item No: : NA & \multicolumn{2}{|l|}{ Manufacturer: } & \multicolumn{2}{|c|}{ Supplier: } \\
\hline \multicolumn{2}{|l|}{ Mig. Part/Model No:: } & \multicolumn{3}{|l|}{ Supplier's P/N: } \\
\hline \multicolumn{5}{|l|}{ Part Description: } \\
\hline \multicolumn{5}{|l|}{ End Use Description: } \\
\hline \multicolumn{5}{|c|}{ Section 2 a Component Information } \\
\hline $\begin{array}{l}\text { Equipment No:: } \\
\text { SCHe-TK-5*01 with } \\
\text { SCHe-V-*114 } \\
\text { SChe-TK-5*02 with } \\
\text { SCHe-V-*115 } \\
\text { SCHe-TK-5*03 with } \\
\text { SCHe-V-*116 } \\
\text { SCHe-TK-5*04 with } \\
\text { SCHE-V-* } 117\end{array}$ & $\begin{array}{l}\text { Specification No: } \\
\text { W-441-P5, Rev. } 2\end{array}$ & $\begin{array}{l}\text { Manufacturer: } \\
\text { Matheson }\end{array}$ & & Past P.O. No: \\
\hline $\begin{array}{l}\text { Procurement and/or } \\
\text { Model No.: Bottle: } \\
\text { Matheson 1A. Valve: } \\
\text { CGA No. } 580\end{array}$ & Equipment Supplier (if c & from manufacturer): & TBD & $\begin{array}{l}\text { Equip. Supplier's Part No.: } \\
\text { NA }\end{array}$ \\
\hline
\end{tabular}

Component Description: These gas bottles and associated valves provide the safety grade helium source for emergency MCO purge.

Section $2 b$ Commercial Availability of the item

1. Is the Item available from a catalogue of a qualified NQA1 supplier? (coordinate with project CGl interface Engineer or BTR)

( 1 YES (go to \#2 below)

[X] NO (go to procedure step 63.2, proceed to dedicate Item.)

If not available from a qualified NQA1 supplier, is it available from an ISO 9000 supplier? (coordinate with project CGI interface Engineer or BTR)

[ ] YES (go to \#2 below, then go to procedure step 6.3.2, proceed to dedicate item)

[ $X$ ] NO (go to procedure step 6.3.2, proceed to dedicate Item.)

2. List of Candidate qualified suppliers or ISO 9000 suppliers company name \& type

contact name

phone

NA

3. Recommended Procurement Strategy (coordinate with project CG1 interface Engineer or BTR):

The bottles and associated valves will be procured as commercial grade. A CGI Upgrade Dedication Form will be prepared. Bottle is steel and is size "1A". Bottles will be fabricated to requirements of DOT standards (49 CFR 178.37). Valves are furnished as part of the helium bottles. The Documentation supplied by the fabricator will include serial number identification for the bottles.

The bottles are not individually seismically tested. The facility equipment rack/restraints holding them require seismic design and certification. Seismic testing of the assembled panel will be done with a "1A" bottle and connecting flex hose installed. 


\begin{tabular}{l} 
Commercial Grade Item Upgrade Dedication Form \\
\begin{tabular}{|l|l|}
\hline ECN No. NA SNI-4887. Kev. 0 \\
Title: SCHe HELIUM SUPPLY BOTTLES AND ASSOCIATED ISOLATION \\
VALVES
\end{tabular} \\
\hline
\end{tabular}

The DOT fabrication report provided by the fabricator will include, but not limited to documentation for: date of fabrication, liquid penetrant certification report, verification of leak test (stem, seat, and rupture disc) of the isolation valves, and hydrotest report.

Section $2 c$ CGI Determination

1. Question \#1: Is the Item subject to design or specification requirements that are unique to nuclear facilities or activities?

l ] YES (the ltem is not commercial grade)

[X] NO (continue)

2. Question \#2: Is the Item used in applications other than nuclear facilities or activities?

l ] NO (the item is not commercial grade)

[X] YES (continue)

3. Question \#3: Is the Item ordered from manufacturer/supplier on the basis or specifications set forth in the published product information (e.g. manufacturer's catalog)?

I I NO (the ltem is not commercial grade)

[X] YES (continue)

[X] All three criteria have been satisfied. The Item meets the definition of commercial grade.

Section $2 \mathrm{~d}$ Reason for Dedication

The above described Item is being Dedicated for use in the application cited for the following reason(s):

[X ] Item is being purchased from a non ESL manufacturer suppier as commercial grade to be used in a Safety Class application.

[ 1 Item is being purchased from a non ESL manufacturer supplier as commercial grade to be used in a Safety Significant application.

[ ] Item was purchased from a non ESL manufacturer supplier as commerciai grade to be used in a Safety Class application.

1 I Item was purchased from a non ESL manufacturer supplier as commercial grade to be used in a Safety Significant application.

I I Other ('like-for-like', similar, substitution, replacement evaluation)

Section 3 Failure Effects Evaluation

A. Part/Component Safety Function:

1. SCHe Pressure Boundary Integrity- Prevents helium leakage from the SCHe System.

2. Maintain critical function before and after Seismic event.

3. Supply Helium for MCO emergency helium purge.

B. Part/Component Functional Mode:

Safety Function \#1:

l ] Active - Mechanical or Electrical change of state is required to occur for the component to perform its safety function

[X] Passive - Change of state is not required for the component to perform its safety function Safety Function \#2:

( ) Active - Mechanical or Electrical change of state is required to occur for the component to perform its safety function.

[X] Passive - Change of state is nat required for the component to perform its safety function Safety Function \#3:

( ) Active -Mechanical or Electrical change of state is required to occur for the component to perform its safety function.

[X] Passive - Change of state is not required for the component to perform its safely function 
Commercial Grade Item Upgrade Dedication Form

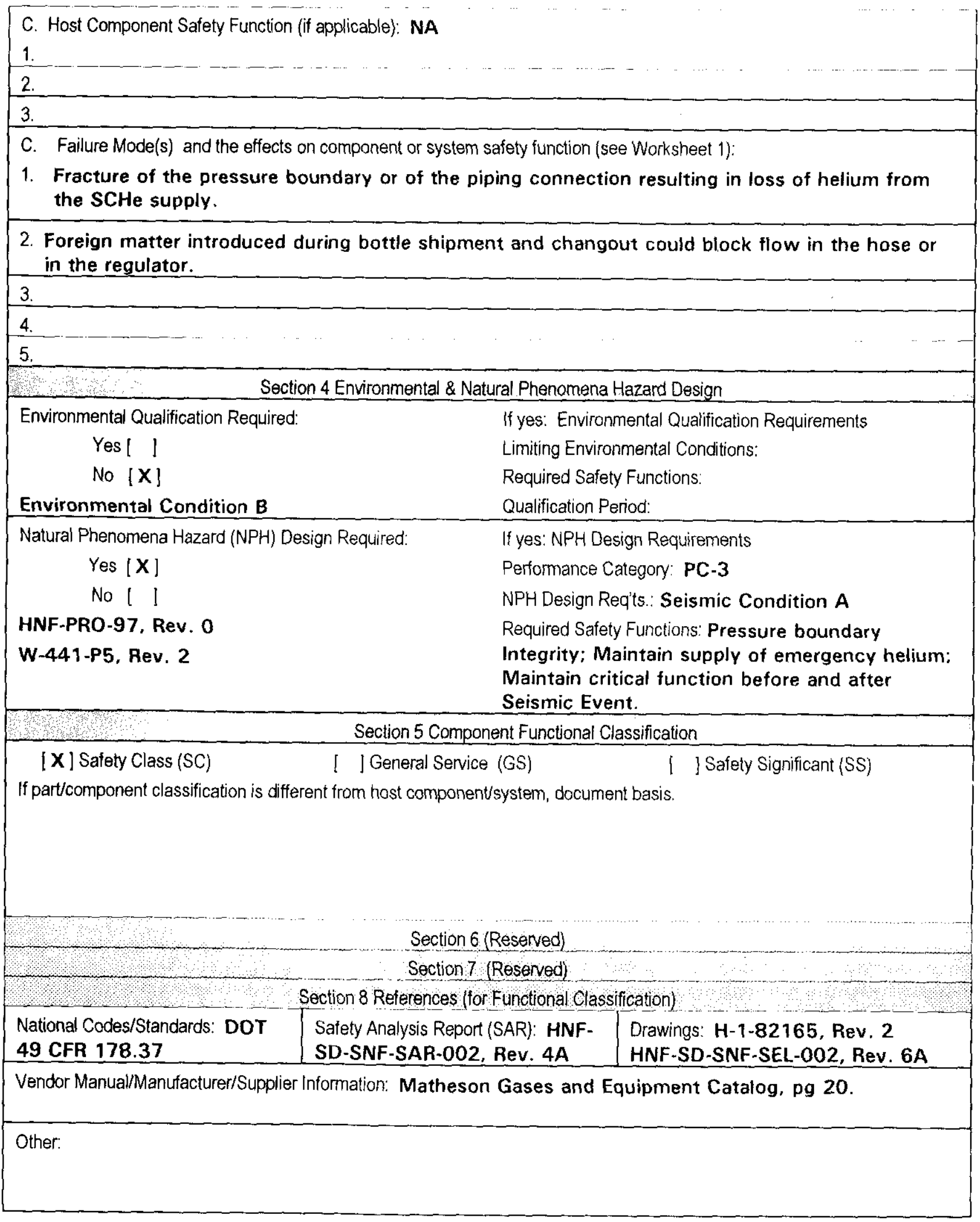


Commercial Grade Item Upgrade Dedication Form

ECN No. NA CGI No. CGI-SNF-D-13-2-P5-050
Title: SCHE HELIUM SUPPLY BOTTLES AND ASSOCIATED ISOLATION
VALVES

Page 4 of 12

\begin{tabular}{|c|c|c|c|c|}
\hline \multicolumn{5}{|c|}{ U $15, \quad$ Section 9 Critical Characteristics } \\
\hline $\begin{array}{l}\text { Critical Characteristics Verification } \\
\text { Document: Vendor Specifications: } \\
\text { HNF-SD-SNF-SEL-002, Rev. 6A }\end{array}$ & Acceptance Criteria/Tolerances & $\begin{array}{l}\text { Acceptance } \\
\text { Method }\end{array}$ & ID & Function \\
\hline \multicolumn{5}{|c|}{ 1. Item Identification Critical Characteristics (necessary for reasonable assurance that the Item delivered is the Item specified) } \\
\hline $\begin{array}{l}\text { Construction Documentation } \\
\text { for DOT } 49 \text { CFR } 178.37\end{array}$ & $\begin{array}{l}\text { Fabrication Report information including: } \\
\text { serial numbers, date of fabrication, liquid } \\
\text { penetrant certification report, verification } \\
\text { of leak test (stem, seat and rupture disc) } \\
\text { of the isolation valves, and hydrotest } \\
\text { report. }\end{array}$ & $1, \mathrm{IN}$ & $x$ & \\
\hline Nameplate - Manufacturer & Matheson & $1, \mathrm{IN}$ & $\mathbf{x}$ & \\
\hline Bottle Size & 1A; 9 inch diameter by 51 inch height & $1, \mathrm{IN}$ & $\underline{x}$ & \\
\hline $\begin{array}{l}\text { Procurement and/or } \\
\text { Model No. }\end{array}$ & $\begin{array}{l}\text { Bottle: Matheson 1A. Valve: CGA No. } \\
580\end{array}$ & $1, \mathrm{IN}$ & $\mathrm{x}$ & \\
\hline \multicolumn{5}{|c|}{ 2. Physical Critical Characteristics (for reasonable assurance that the Item delivered is the Item specified) } \\
\hline Material, Bottle & Steel (Note 3) & $1 . \mathrm{IN}$ & $\mathrm{x}$ & \\
\hline Material, Process Connection & Stainless Steel (Note 3) & $\begin{array}{l}1, \mathrm{IN} \\
1 . \mathrm{T}\end{array}$ & $x$ & \\
\hline & & & & \\
\hline & & & & \\
\hline & & & & \\
\hline
\end{tabular}

\begin{tabular}{|l|l|l|l|}
\hline 3 Performance Critical Characteristics (for reasonable assurance that the item will perfom its intended safety function(s) \\
\hline Pressure Boundary Integrity & $\begin{array}{l}\text { Pressure Test at minimum 3300 psig (See } \\
\text { Fabrication Report Hydrotest } \\
\text { Documentation) }\end{array}$ & $\mathbf{X}$ \\
\hline Environmental & Note 1 & & \\
\hline Seismic Condition A Event & Note 2 & 1, T & X \\
\hline $\begin{array}{l}\text { Valve Leakage, Stem, Seat, } \\
\text { and Rupture Disc. }\end{array}$ & $\begin{array}{l}\text { Pressure Test at 3300 psig (No Bubbles) } \\
\text { See Fabrication Report }\end{array}$ & 1, N & $\mathbf{X}$ \\
\hline
\end{tabular}

4. Notes and Legend:

1. The helium bottle and valve are not subject to degradation at ambient conditions of $40^{\circ} \mathrm{F}$ and $60 \% \mathrm{RH}$ or $115^{\circ} \mathrm{F}$ and $22 \% \mathrm{RH}$ and is suitable for Environmental Condition B application.

2. Maintain critical function before and after Seismic event. $W$ 441-P5, Rev. 2, Appendix I, page 1-2, provides a seismic testing plan for these components at a seismic spectra TBD.

Equipment that has been shaker-table tested should not be installed in a plant (Ref. IEEE Standard 344-1984, Section 7). Consequently, the seismic test constitutes a destructive test.

Acceptance Method:

1. Special Test and Inspection

1, IN for Inspection

1,T Tor Test

2. Commercial Grade Survey

3. Source Verification

4. Vendor/ltem History

3. Material verification acceptance method may be by either inspection or test. 
Commercial Grade Item Upgrade Dedication Form ECN No. NA COI No. CGI-SNF-D-13-2-P5-050 SNF-4X87. ReN. 0

Title: SCHe HELIUM SUPPLY BOTTLES AND ASSOCIATED ISOLATION VALVES

Section 10 Initial Review and Approval

Approvals:

Designated Engineer:

Design Authority:

cosh

QA Engineer: $5 \times 20141 / 27 / 99$ 
Commercial Grade Item Upgrade Dedication Form

ECN No. NA __ CGI No. CGI-SNF-D-13-2-P5-050

SNl-4887. Rev. O

Title: SCHE HELIUM SUPPLY BOTTLES AND ASSOCIATED ISOLATION

VALVES

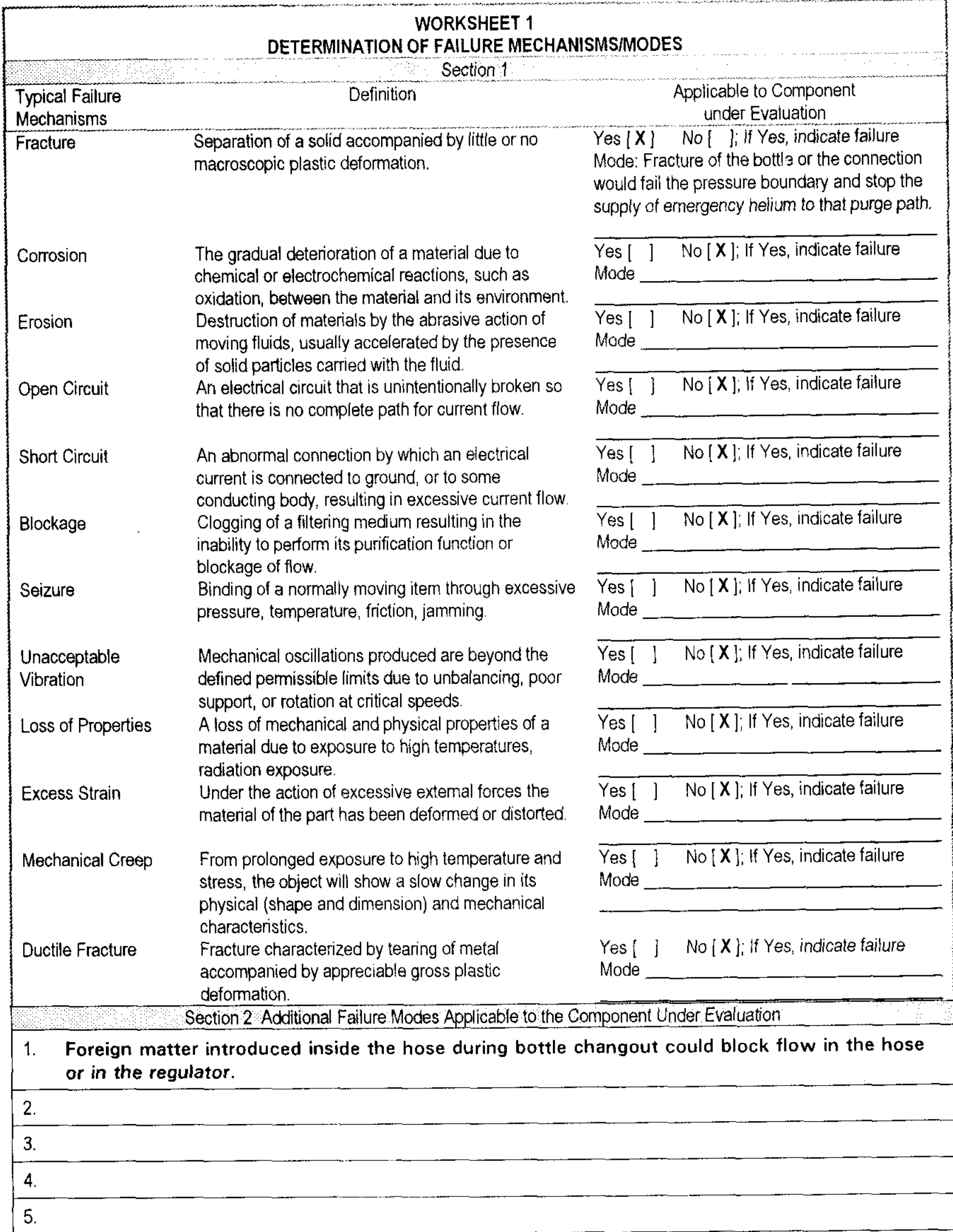


Commercial Grade Item Upgrade Dedication Form

ECN No. NA CGI No. CGI-SNF-D-13-2-P5-050

Title: SCHe HELIUM SUPPLY BOTTLES AND ASSOCIATED ISOLATION

VALVES

\section{CHECKLIST 1}

ACCEP'TANCE METHOD 1

SPECIAL TEST/INSPECTION VERIFICATION

\begin{tabular}{|c|c|c|c|c|}
\hline & & & & SECTION 1 \\
\hline $\begin{array}{l}\text { Item } L \\
\text { and } A \\
\text { Systen }\end{array}$ & $\begin{array}{l}\text { escrip } \\
\text { ssocia } \\
\text { \#: } 1\end{array}$ & $\begin{array}{l}\text { on: SC } \\
\text { ted } \mathrm{Va} \\
-2\end{array}$ & $\begin{array}{l}\text { He Helium Supply Bottles } \\
\text { ves }\end{array}$ & $\begin{array}{l}\text { Equip \#: SCHe-TK-5*01,5*02,5*03,5*04 with } \\
\text { valves SCHe-V-*114, *115, }{ }^{*} 116 \text { and }{ }^{*} 117 \\
\text { respectively. } \\
\text { Procurcment and/or Model No.: Bottle: Matheson } 1 A . \\
\text { Valve: CGA No. } 580\end{array}$ \\
\hline $\begin{array}{l}\text { Math } \\
6775 \\
\text { Newa } \\
\text { P.O. \# }\end{array}$ & $\begin{array}{r}\text { Mal } \\
\text { eson G } \\
\text { Centr } \\
\text { rk, CA }\end{array}$ & $\begin{array}{l}\text { ufactur } \\
\text { as Pro } \\
\text { Ave. } \\
9456\end{array}$ & $\begin{array}{l}\text { (Address/Phone): } \\
\text { lucts }\end{array}$ & Supplier (Address/Phone): \\
\hline & & & SECTION 2 CRITICAL CHARAC & TERISTICS TO BE VERIFIED BY METHOD 1 . \\
\hline & Test & $\begin{array}{l}\text { Post- } \\
\text { Test }\end{array}$ & & \\
\hline$[\mathbf{X}]$ & [ l & [ l & 1. Construction Docum & ntation \\
\hline$[\mathbf{X}]$ & {$[1$} & 11 & 2. Nameplate - Manufa & turer \\
\hline$[\mathbf{X}]$ & [ ] & {$[1]$} & 3. Bottle Size & \\
\hline$[\mathbf{X}]$ & l 1 & 1 1 & Procurement and/or & Model No. \\
\hline$[\mathbf{X}]$ & {$[\mathbf{X}]$} & 1 I & 5. Material, Bottle (Ver & ication may be by either inspection or test) \\
\hline$[\mathbf{X}]$ & {$[\mathbf{X}]$} & I I & $\begin{array}{l}\text { 6. Material, Process Co } \\
\text { test) }\end{array}$ & inection (Verification may be by either inspection or \\
\hline$[\mathbf{X}]$ & {$[$ ] } & [ ] & 7. Pressure Boundary II & tegrity (See Fabrication Report) \\
\hline [ l & {$[\mathbf{X}]$} & [ ] & 8. Seismic Condition A & \\
\hline$[\mathbf{X}]$ & [ ] & [ ] & 9. Valve Leakage (See & abrication Report) \\
\hline & & & SECTION 3 & ERIFIED BY INSPECTION \\
\hline * See & Attachir & eent $\mathrm{H}$ & f Desk Instniction for Sampli & gize \\
\hline Chara & teristic & : Cons & truction Documentation & \\
\hline Sampl & Size* & All It & & \\
\hline $\begin{array}{l}\text { Accep } \\
\text { certif } \\
\text { hydro }\end{array}$ & $\begin{array}{l}\text { ance } C \\
\text { cation } \\
\text { test c }\end{array}$ & $\begin{array}{l}\text { ritcria: } \\
\text { verifi } \\
\text { rtifica }\end{array}$ & $\begin{array}{l}\text { Fabrication report includin } \\
\text { ation of leak test (stem, s } \\
\text { ion. }\end{array}$ & $\begin{array}{l}\text { : serial numbers, date of fabrication, liquid penetrant } \\
\text { at, and rupture disc) of the isolation valves, and }\end{array}$ \\
\hline Recei & t Inspe & ction PI & In / Report \#: & - \\
\hline Refer & nces ( & ee Secti & n): & \\
\hline
\end{tabular}


Characteristic: Nameplate - Manufacturer

Sample Size*: All Items

Acceptance Criteria: Matheson

Receipt Inspection Plan / Report \#:

References (see Section 8): Matheson Gases and Equipment Catalog, pg

20.

Characteristic: Bottle Size

Sample Size*: All Items

Acceptance Criteria: 1A; 9 inch diameter by 51 inch height

Receipt Inspection Plan / Report \#:

References (see Section 8):

Characteristic: Procurement and/or Model No.

Sample Size*: All Items

Acceptance Criteria: Bottle: Matheson 1A. Valve: CGA No. 580

Receipt Inspection Plan / Report \#:

References (see Section 8):

Characteristic: Material, Bottle

Sample Size*: All Items

Acceptance Criteria: Steel

Receipt Inspection Plan / Report \#:

References (see Section 8):

Characteristic: Material, Process Connection

Sample Size*: All Items

Acceptance Criteria: Stainless Steel

Receipt Inspection Plan / Report \#:

References (see Section 8):

Characteristic:

Sample Size*: All Items

Acceptance Criteria:

Receipt Inspection Plan / Report \#:

References (see Section 8): 


\section{SECTION 4 VERIFIED BY SPECIAL TEST}

* See Attachment $\mathrm{H}$ of Desk Instruction for Sampling Size

Test To Be Performed by:

Number of ltems to be Tested:

[ ] Purchaser

I ] Supplier/Manufacturer**

[ ] Other

Characteristic for Test: Pressure Boundary Integrity

Acceptance Criteria: Minimum Test Pressure of 3300 psig (110\% of 3000 psig system design)

Sample Size*: Normal Sampling Size

Actual Test Value:

Test Plan and Report \#: References (see Section 8): See Fabrication Report Hydrotest Documentation

Characteristic for Test: Seismic Condition A

Acceptance Criteria: Maintain Critical Function Before And After Seismic Event. W-441-P5, Rev. 2. Appendix I, page 1-2, provides a seismic testing plan for these components at a seismic spectra TBD Sample Size*: W-441-P5, Rev. 2, Appendix I, page 1-2, provides the seismic testing plan for these components. The seismic testing is conducted for one complete panel with the components assembled on the panel and tested as a complete assembly. The test seismically qualifies the entire assembly, including mountings, piping, and components. The number of components tested is dictated by the panel assembly design.

Actual Test Value:

Test Plan and Report \#: References (see Section 8):

Characteristic for Test: Valve Leakage, (stem, seat, and rupture disc)

Acceptance Criteria: No Bubbles at Test Pressure of 3300 psig

Sample Size*: Normal Sampling Size

Actual Test Value:

Test Plan and Report \#: References (sce Scction 8): See Fabrication

Report

Characteristic for Test:

Acceptance Criteria:

Sample Size*: Normal Sampling Size

Actual Test Value:

Test Plan and Report \#: References (sec Section 8):

Characteristic for Test:

Acceptance Criteria:

Sample Size*: Normal Sampling Size

Actual Test Value:

Test Plan and Report \#: References (see Section 8):

** If Supplier/Manufacturer or Other, Refer to C(il Checklist-2 for Support Information 
Commercial Grade Item Upgrade Dedication Form

ECN No. NA _- CGI No. CGI-SNF-D-13-2-P5-050 SNF-4887, Rev. 0

Title: SCHe HELIUM SUPPLY BOTTLES AND ASSOCIATED ISOLATION

VALVES

SECTION 5 TEST IINSPECTION SUMMARY (ACceptance Method 1)

\begin{tabular}{|c|c|c|c|c|c|c|c|c|c|c|c|}
\hline \multicolumn{12}{|c|}{ SECTION 5 TEST I INSPECTION SUMMARY (Acceptance Method 1 ). } \\
\hline \multicolumn{12}{|c|}{ 1. SUMMARY OF VERIFIED CRITICAL CHARACTERISTICS, THEIR VERIFICATION METHODS, AND RESULTS } \\
\hline \multicolumn{12}{|c|}{ ITEM DESCRIPTION: } \\
\hline \multicolumn{4}{|c|}{ Critical Characteristics } & \multicolumn{8}{|c|}{ Verification Results } \\
\hline Crtical Characteristics & Acceptance Criteria Tolerances & ID & Function & $\begin{array}{c}\text { Method } \\
\text { TIN }\end{array}$ & $\begin{array}{c}\text { Procedure } \\
\text { or RR } \#\end{array}$ & $\begin{array}{l}\text { Check- } \\
\text { list ID }\end{array}$ & $\begin{array}{c}\text { Number } \\
\text { Tested }\end{array}$ & $\begin{array}{l}\text { Number } \\
\text { Failed } \\
\end{array}$ & $\begin{array}{c}\text { Verifying } \\
\text { Organization }\end{array}$ & Printed Name Signature & Date \\
\hline $\begin{array}{l}\text { Construction } \\
\text { Documentation }\end{array}$ & $\begin{array}{l}\text { Fabrication Report } \\
\text { information including: } \\
\text { serial numbers, date of } \\
\text { fabrication, liquid } \\
\text { penetrant certification } \\
\text { report, verification of } \\
\text { leak test (stem, seat, } \\
\text { and rupture disc) of the } \\
\text { isolation valves, and } \\
\text { hydrotest report. }\end{array}$ & $x$ & & & & & & & & & \\
\hline $\begin{array}{l}\text { Nameplate - } \\
\text { Manufacturer }\end{array}$ & Matheson & $\mathrm{x}$ & & & & & & & & & \\
\hline Bottle Size & $\begin{array}{l}1 A ; 9 \text { inch diameter } x \\
51 \text { inch height }\end{array}$ & $\mathrm{x}$ & & & & & & & & & \\
\hline $\begin{array}{l}\text { Procurement and/or } \\
\text { Model No. }\end{array}$ & $\begin{array}{l}\text { Bottle: Matheson } 1 \mathrm{~A} . \\
\text { Valve: CGA No. } 580\end{array}$ & $\mathrm{x}$ & & & & & & & & & \\
\hline Material, Bottle & Steel & $x$ & & & & & & & & & \\
\hline $\begin{array}{l}\text { Material, Process } \\
\text { Connection }\end{array}$ & Stainless Steel & $x$ & & & & & & & & & \\
\hline $\begin{array}{l}\text { Pressure Boundary } \\
\text { Integrity }\end{array}$ & $\begin{array}{l}\text { Pressure Test at } \\
\text { minimum } 3300 \text { psig } \\
\text { (See Fabrication Report } \\
\text { Hydrotest } \\
\text { Documentation) }\end{array}$ & & $x$ & & & & & & & & \\
\hline Seismic Condition A & $\begin{array}{l}\text { Maintain critical function } \\
\text { before and after Seismic } \\
\text { Event }\end{array}$ & & $x$ & & & & & & & & \\
\hline
\end{tabular}


Commercial Grade Item Upgrade Dedication Form

Title: SCHe HELIUM SUPPLY BOTTLES AND ASSOCIATED ISOLATION

VALVES

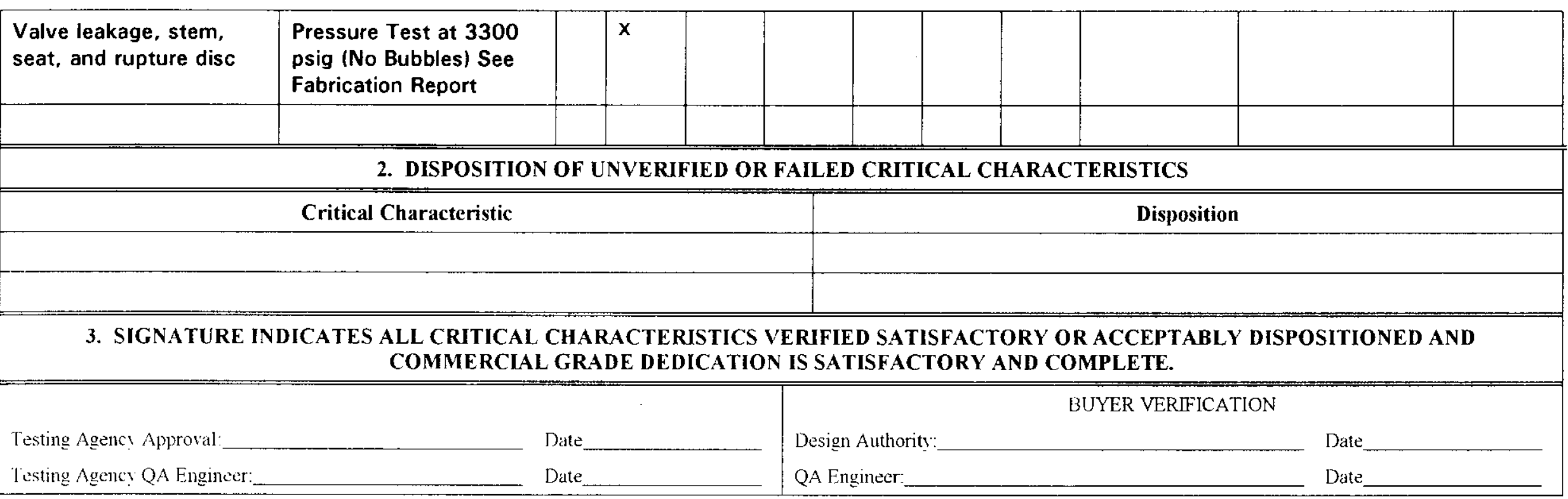


Commercial Grade Item Upgrade Dedication Form

Title: SCHe HELIUM SUPPLY BOTTLES AND ASSOCIATED ISOLATION

VALVES

\section{SECTION 6 CONTACTS/PHONE NUMBERS}

Nane

Phone

Design Authority

QA

QC

Cog - Engineer

CGI Engineer

Procurement Engineer

Other

\section{SECTION 7 SUPPORTING DOCUMENTATION FOR THIS CHECKLIST}

\begin{tabular}{|c|c|}
\hline Initial Procurement Documents & For Critical Characteristics \\
\hline I ] Drawings: & \\
\hline [ I Manuals (specify type \& number): & \\
\hline [ ] Design Calculations & \\
\hline [ ] Installation Instructions & \\
\hline I l Operation Instructions & \\
\hline I I Calibration Instructions & \\
\hline I ] Manufacturer's Recommended Spare Parts List & \\
\hline [ ] Other: & \\
\hline Procurement Documents & \\
\hline [ I Certificate of Conformance/Compliance & \\
\hline [ ] Seismic Qualification Certificatc & \\
\hline I I Environmental Qualification Certificate & \\
\hline [ ] Test Report (s): & \\
\hline I I Inspection Report (s): & \\
\hline [ ] CMTRs for ASME Pressure Rctaining Materials & \\
\hline I ] Valve Seat Leakage Report & \\
\hline [ ] Weld Records & \\
\hline [ ] Material Traceability Record & \\
\hline I ] Other: & \\
\hline
\end{tabular}




\section{DISTRIBUTION SHEET}

\begin{tabular}{|l|l}
\hline To & From \\
Distribution & SNE-CVD \\
\hline
\end{tabular}

\section{Project TitleN Work Order}

W-441, SNF-4887, SCHe Helium Supply Bottles and Associated Valves
Page 1 of 1

Date $7 / 22 / 99$

EDT No. 626322

ECN No. N/A

\begin{tabular}{|c|c|c|c|c|c|}
\hline Name & MSIN & $\begin{array}{l}\text { Text } \\
\text { With All } \\
\text { Attach. }\end{array}$ & Text Only & $\begin{array}{l}\text { Attach./ } \\
\text { Appendix } \\
\text { Only }\end{array}$ & $\begin{array}{c}\text { EDT/ECN } \\
\text { Only }\end{array}$ \\
\hline C. Miska & $\mathrm{R} 3-86$ & $x$ & & & \\
\hline C. Van Katwijk & $\mathrm{R} 3-47$ & $x$ & & & \\
\hline K. Boes (CVD Library) & $\mathrm{R} 3-86$ & $x$ & & & \\
\hline Rom $1 / 27 / 94$ R. Ramsgate & $\mathrm{R} 3-86$ & $x$ & & & \\
\hline J. Brehm & $R 3-26$ & $x$ & & & \\
\hline D. Whitworth & R3-1i & $\mathrm{x}$ & & & \\
\hline P. Morrell (AVS) & $\mathrm{G} 1-50$ & $\mathrm{x}$ & & & \\
\hline M. Evarts (AI) & $\mathrm{N} 1-29$ & $x$ & & & \\
\hline D. Marburger & $\mathrm{R} 3-47$ & $x$ & & & \\
\hline S. Mischke & $23-86$ & $x$ & & & \\
\hline SNF STARIUP & $B 2-64$ & $x$ & & & \\
\hline & & & & & \\
\hline & & & & & \\
\hline & & & & & \\
\hline & & & & & \\
\hline & & & & & \\
\hline & & & & & \\
\hline & & & & & \\
\hline & & & & & \\
\hline & & & & & \\
\hline & & & & & \\
\hline & & & & & \\
\hline & & & & & \\
\hline & & & & & \\
\hline & & & & & \\
\hline & & & & & \\
\hline & & & & & \\
\hline & & & & & \\
\hline
\end{tabular}

